\title{
Extended endoscopic mucosal resection in the esophagus and hypopharynx: a new rigid device
}

\author{
Yves Jaquet • Raphaelle Pilloud • Pierre Grosjean • \\ Alexandre Radu $\cdot$ Philippe Monnier
}

Received: 11 April 2006 / Accepted: 21 August 2006 / Published online: 17 October 2006

(C) Springer-Verlag 2006

\begin{abstract}
We present a new device allowing for the diagnosis and treatment of extended superficial lesions of the esophagus and hypopharynx such as early squamous cell carcinoma, intestinal metaplasia with high grade intraepithelial neoplasia or early adenocarcinoma arising in Barrett's esophagus. A new modified rigid esophagoscope (Karl Storz GmbH, Germany) has been designed. A large mucosal area is sucked against a transparent and perforated hemi-cylindrical window. Mucosal resection is performed by an electrical wire loop at a constant depth of $1 \pm 0.1 \mathrm{~mm}$. The resected surface varies from 4 to $12 \mathrm{~cm}^{2}$. Circumferential resection consists of two opposite individual hemi-circumferential resections. We performed three series of animal trials: hemicircumferential mucosectomies; circumferential resections of variable $(2$ to $6 \mathrm{~cm}$ ) length and long-segment mucosectomies with follow-up. Hemi- and circumferential resections could be done in one or two specimens only which allowed precise histological studies. This facilitated easy orientation and analysis of the surgical margins. The deep resection margin was precisely located at the submucosal level, a prerequisite for a safe resection of superficial cancers of the esophagus and hypopharynx.
\end{abstract}

Keywords Pharynx - Esophagus - Early cancer · Barrett's esophagus $\cdot$ Minimally invasive surgery

\footnotetext{
Y. Jaquet $(\bowtie) \cdot$ R. Pilloud · P. Grosjean ·

A. Radu $\cdot$ P. Monnier

Department of Otolaryngology,

Head and Neck surgery,

Centre Hospitalier Universitaire Vaudois,

CH-1011 Lausanne, Switzerland

e-mail: yves.jaquet@chuv.ch
}

\section{Introduction}

There is a growing interest to treat early superficial lesions of the upper digestive tract with minimally invasive techniques. Diseases such as early squamous cell carcinoma (SCC) of the pharynx and esophagus, Barrett's esophagus (BE) with high grade intraepithelial neoplasia (HGIN) or early adenocarcinoma (AC) are now within the scope of endoscopic treatment modalities.

In the esophagus, the rate of lymph node metastasis is limited for tumors invading the lamina propria and muscularis mucosae, but increases rapidly as the tumor invades the submucosa (Fig. 1) [2]. Consequently, only patients with $\mathrm{T} 1 \mathrm{a}$ or $\mathrm{m} 1$ to $\mathrm{m} 3$ lesions are good candidates for endoscopic mucosal resection (EMR) [2].

Endoscopic mucosal resection (EMR) in the esophagus with the gastrofiberscope is limited to focal lesions as single mucosal resections do not exceed $2 \mathrm{~cm}^{2}$ with the cap-fitted endoscope. Attempts at removing widespread early SCC or the whole metaplastic mucosa of circumferential BE with the flexible endoscope demonstrated that piecemeal resections took a long time to be completed and often required multiple sessions of treatments $[15,17]$. This has stimulated the gastroenterologic community to develop new techniques for "en bloc" resection of larger mucosal specimens with needle-knives of different kinds (insulated-tip, triangle-tip knife and sheated knife) $[3,13,14]$. Whatever the technique, the only rational treatment for multicentric foci of early SCC or early AC arising in BE is the entire resection of the carcinomatous lesion or of the columnar-lined segment. Indeed, in BE, complete removal of the metaplastic mucosa will simultaneously eliminate all foci of dysplasia or early cancer potentially missed by random biopsies [8]. 
T1a

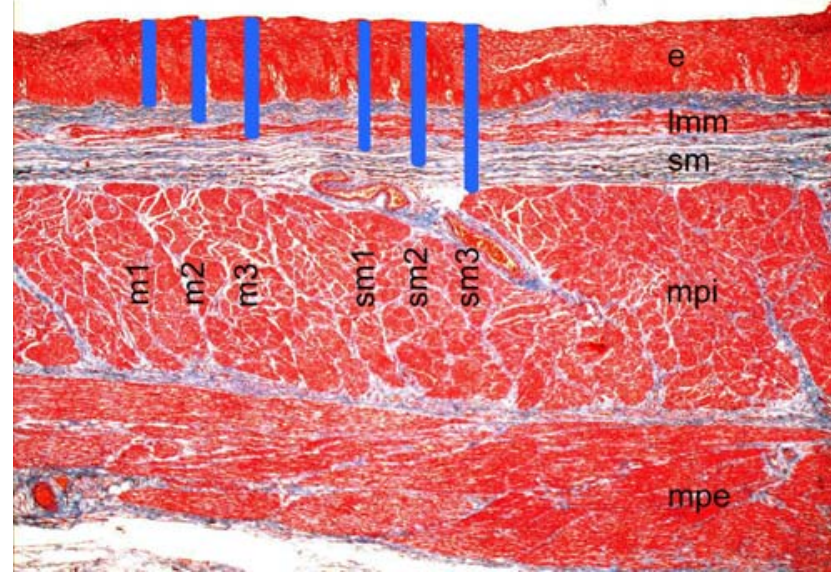

Fig. 1 Classifications of early esophageal carcinoma. T1a: intramucosal carcinoma (not infiltrating beyond the muscularis mucosae): $m 1$ : intraepithelial tumors, $m 2$ : tumors invading the lamina propria, $m 3$ : tumors in contact with or invading the lamina muscularis mucosae. $T 1 b$ : submucosal carcinoma: $s m 1$ : tumors invading the most superficial two thirds of the submucosa, $s m 2$ : tumors invading the most superficial two thirds of the submucosa, sm3: tumors invading the deep third of the submucosa. According to Eguchi et al. [2], lymph node metastases are found in $0 \%$ of $m 1$ cancers, $5.6 \%$ of $m 2,10.3 \%$ of $m 3$ without lymphatic invasion and $41 \%$ of $m 3$ with lymphatic involvement. The rate of lymph node metastasis for submucosal cancers reaches up to $80 \%$. Only intramucosal ( $T 1 a)$ cancers can thus be considered as "early cancers"

Head and neck cancer patients are at high risk for the development of second primary malignancies of the upper aero-digestive tract. The use of routine panendoscopy at the time of diagnosis $[5,19]$ and abrasive cytology [10] during follow-up has led to a high rate of detection of second primaries. Most of these tumors are diagnosed at an early stage ( $>90 \%$ in situ or microinvasive carcinomas) and these patients can be cured by minimally invasive endoscopic resection.

The aim of our studies was the development of a new rigid instrument intended to treat superficial cancerous or precancerous conditions of different sizes in the hypopharynx and esophagus.

\section{Materials and methods}

The principle of endoscopic mucosal resection with the rigid endoscope relies on the aspiration of the mucosa against a flat surface followed by its precise cut at a regular depth with an electrocautery device $[4,11,12]$.

The modified esophagoscope (Fig. 2) (KARL STORZ GmbH \& Co, Tüttlingen, Germany) is made up of a metallic outer tube of $15 \mathrm{~mm}$ in diameter at the distal end of which a lateral aperture of $6 \mathrm{~cm}$ in length with an opening of $200^{\circ}$ is designed (Fig. 2a). The introduction into the esophagus is carried out under visual control with a $0^{\circ}$ telescope inserted through the outer tube. This seals its distal aperture in order to avoid potential mucosal tears during diagnostic endoscopies. Once at the level of the lesion, the $0^{\circ}$ telescope is removed and replaced by the resectoscope (Fig. 2b, c). Its extremity consists of a transparent and perforated window and a diathermy wire loop that can be moved through the window (Fig. 2d). An angulated $30^{\circ}$ telescope placed inside the resectoscope allows side viewing through the window opened at $180^{\circ}$. The resection margins can be controlled by rotating the telescope at $90^{\circ}$ on both sides (Fig. 2e). This allows the accurate targeting of the lesion. When suction is turned on, the negative pressure applied inside the resectoscope aspirates the mucosa and part of the submucosa against the perforated window. The wire loop is connected to a high-frequency power device (Erbe-Tom T400 ${ }^{\circledR}$ ) initiated by a foot pedal. The section of the electric wire loop is of $0.3 \mathrm{~mm}$ in diameter and induces only minimal thermal damage. The depth of resection is determined by the constant distance of $1.0 \pm 0.1 \mathrm{~mm}$ between the wire loop and the transparent window. The surgeon must perform the resection at a constant speed (about $1 \mathrm{~cm} / \mathrm{s}$ ) to avoid mucosal tears. Once the lesion has been targeted and sucked against the transparent window, the resection is performed in less than $15 \mathrm{~s}$. The resected piece of mucosa remains sucked against the transparent window and can thus be removed together with the endoscope. The specimen is then stretched to its original size and fixed in $4 \%$ Formalin for histological examination.

Considering the size of the lesion and the needed surgical security margins, a window length of $2-6 \mathrm{~cm}$ can be utilized. The width of the resected area, defined by the hemi-circumferential aperture of the window, is of $2 \pm 0.2 \mathrm{~cm}$. A piece of mucosa of up to $12 \mathrm{~cm}^{2}$ can thus be obtained as a single resected specimen. The depth of resection, chosen for a precise cut throughout the submucosa of the esophagus, includes the lamina muscularis mucosae without damage to the muscularis propria (Fig. 3). This condition warrants the complete ablation of the diseased mucosa as well as early cancers reaching down to the lamina muscularis mucosae.

\section{Results}

The experimental development of the rigid esophagoscope required an appropriate animal model. The sheep esophagus is very similar to that of humans with 
Fig. 2 modified rigid esophagoscope for mucosectomy. a Outer tube. b Resectoscope with wire loop and cartridge. c EMR esophagoscope with resectoscope. d Close-up view of the cartridge, transparent perforated window and electric wire loop. e Rotation of the telescope on both sides to control lateral section margins
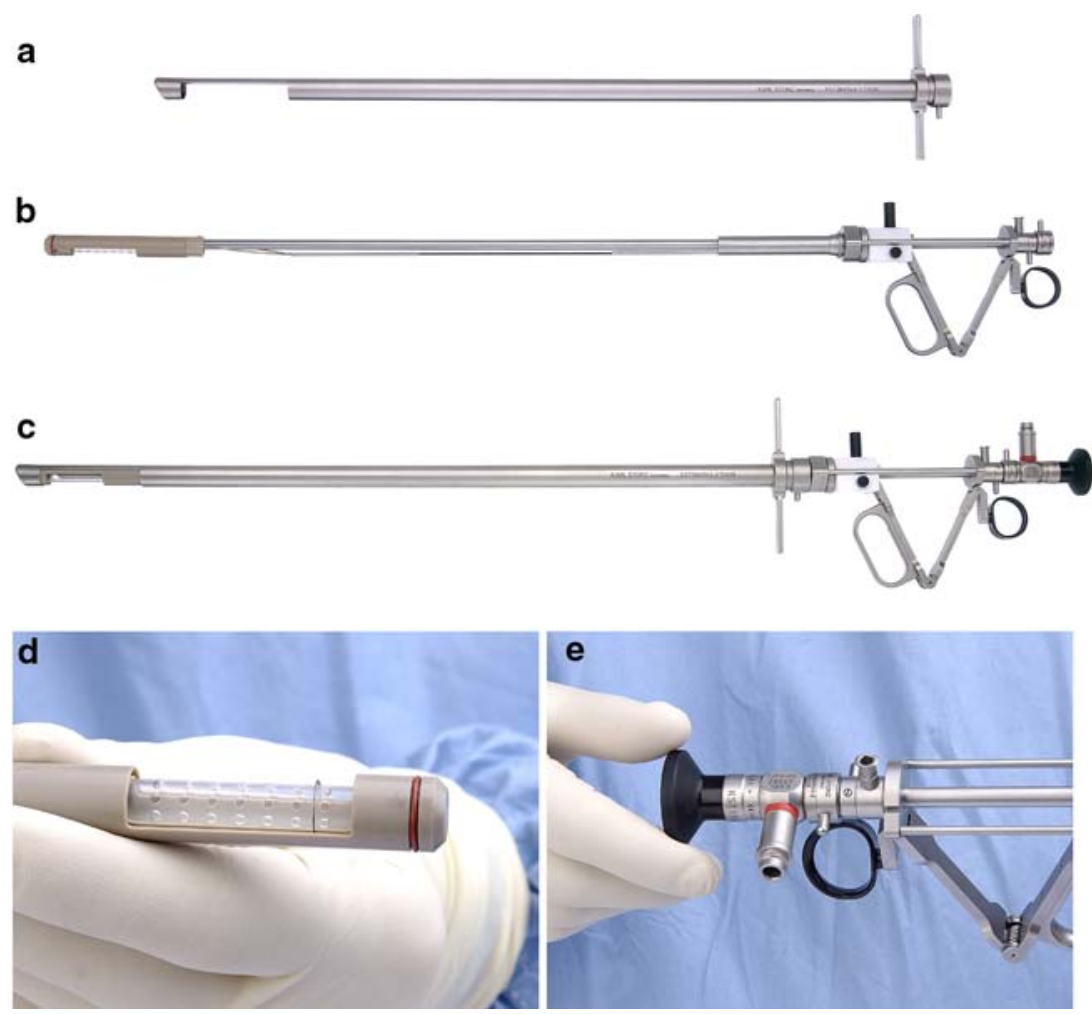

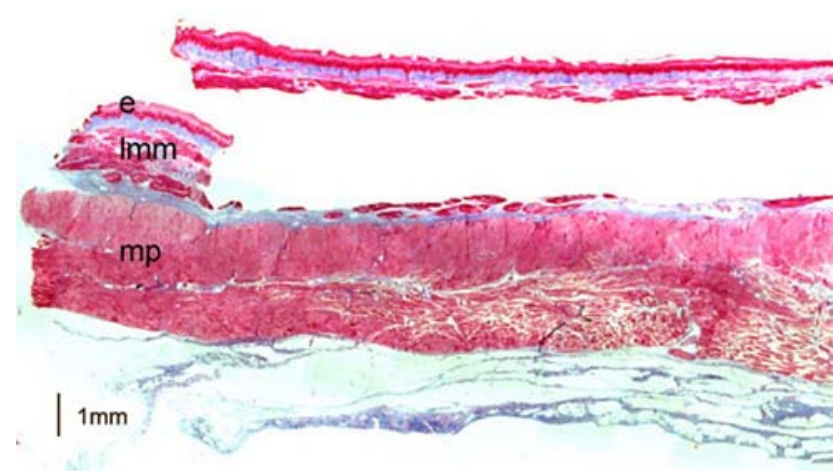

Fig. 3 Resected specimen and corresponding esophageal wall. Regular section performed throughout the submucosa. $E$ epithelium, $L M M$ lamina muscularis mucosae, $M P$ muscularis propria (masson trichrome staining)

respect to the histological organization of its wall and the thickness of the separate layers.

Grosjean et al. [4] carried out the first eight hemicircumferential resections in five sheep and demonstrated that a constant depth of resection was achieved over the whole length of the resected specimen. No perforation occurred and all animals healed without cicatricial stenosis. This study helped define several parameters such as the diameter and shape of the resection wire, and the intensity and frequency of the electrical power used. A further modified device was subsequently used by Radu et al. [12]. They performed 55 additional hemi-circumferential EMRs in the sheep and obtained 48/55 (87\%) accurate resections throughout the submucosa. In the remaining seven animals, scarce fibers of muscularis propria were found over the resected specimens. However, neither perforation nor excessive bleeding was encountered. All animals showed complete reepithelialization within 3 months and no stenosis was observed.

Recent experimental studies investigated circumferential resections in the esophagus in the hope of treating circumferential Barrett's esophagus in humans in the future $[11,12]$. A $360^{\circ}$ resection was completed by two opposite $180^{\circ}$ resections. Overlap of two resections was avoided by visual control of the aspirated mucosa before the second resection. Healing by secondary intention occurred with wound contraction and growth of granulation tissue leading to varying degrees of cicatricial stenosis. This phenomenon was exacerbated by the superimposed inflammation caused by food passage and gastric reflux, over several weeks. The stenosing process decreased rapidly as soon as the epithelial protection of the muscularis propria was restored.

Since $360^{\circ}$ EMR with the rigid endoscope was feasible, we studied several lengths of circumferential resections from 2.2 to $5.5 \mathrm{~cm}(n=24)$ [11]. Follow-up endoscopies were carried out monthly after the mucosectomy including dilatation by bougienage when cicatricial strictures were observed. The $360^{\circ}$ resections were successfully performed at all lengths. One perforation $(1 / 24)$ occurred during EMR by overlapping the 
two opposite resections. The number of dilatations needed to treat strictures ranged from 1.3 per animal $(2.2 \mathrm{~cm}$ resections) to $2.6(5.5 \mathrm{~cm}$ resections $)$ and increased with the length of resection (Table 1). In $38 \%$ of the cases, no dilatation was required. Only one residual stenosis $(5 \%)$, albeit moderate (11 $\mathrm{mm}$ in diameter), was observed at the end of the follow-up study.

\section{Discussion}

\section{EMR in Barrett's esophagus}

Esophagectomy is still considered as the gold standard for the curative treatment of HGIN or early cancer (Tis, T1a) of the esophagus. However, the morbidity and mortality of this procedure can be significant in elderly patients who are often poor candidates for surgery. The need for minimally invasive procedures to treat early SCC, HGIN, and early AC arising in $\mathrm{BE}$, is also substantiated by the low prevalence $(7 \%)$ of regional lymph node metastases in intramucosal ACs and SCC (Tis and T1a) [8].

Surveillance of BE patients, by random biopsies, has two major disadvantages: a debatable cost-effectiveness and a low negative predictive value [9]. Moreover, the presence of multifocal ACs in resected esophagi with diagnosed BE with HGIN reinforces the necessity of eradicating the whole metaplastic mucosa in order to achieve a curative treatment, and thus alleviate the need for endoscopic follow-up [9]. On the assumption that the mucosal resection should eradicate the disease, the resected specimen(s) should encompass the whole diseased mucosa. Of utmost importance is the accuracy of the histological analysis which implies a precise orientation and a limited number of specimens.

Few authors have investigated circumferential resections in the human esophagus. Seewald et al. [17] treated 12 patients with circumferential segments of BE with HGIN or early cancer. A median number of five sessions were necessary and a median surface of
$3.8 \mathrm{~cm}^{2}$ was removed at each session. Satodate et al. [15] reported one case of circumferential EMR with the flexible endoscope in a single session for a long-segment $\mathrm{BE}$ of $5 \mathrm{~cm}$. More than 20 separate resections were necessary to achieve the complete $360^{\circ}$ mucosectomy. Giovannini et al. [3] performed 21 circumferential EMRs with the flexible scope by stripping large mucosal areas following submucosal injections. Their technique required at least two sessions of treatment and histological examination showed a high rate of incomplete resection. Widespread EMR with the flexible scope was recently reported in six pigs but this technique still needs further appraisal [13]. Many drawbacks are encountered when flexible endoscopes are used for large mucosal resections. The small surface of each separate resection requires fastidious procedures and generally multiple sessions of treatment. The mapping of the numerous resected specimens by the pathologist is hazardous and the interpretation of resection margins often unreliable. In addition, separate resections are not easily adjustable to each other, leading to a high rate of incomplete resections of around $11-60 \%$ [6]. In an attempt at improving these results, Rösch et al. [14] have reported their experience in 37 patients undergoing en bloc EMRs for mucosal and submucosal tumors using the insulated tip-knife. Complete tumor removal was achieved in only $25 \%$ of the mucosal lesions although 21 of 37 (58\%) measured less than $2 \mathrm{~cm}$ in diameter. When considering piecemeal resections, this result improved to $65 \%$. In experienced hands, the procedure lasted for $45 \mathrm{~min}-2 \mathrm{~h}$ and the dissection was almost never precisely carried out in the very same tissue plane. The authors conclude that "we must strive for methods of mucosectomy that are improved, from an oncological standpoint, that is en bloc removal with adequate safety margins".

\section{EMR in head and neck cancer}

Multiple primary malignancies are found in a high percentage of patients with head and neck cancer.

Table 1 Endoscopic follow-up after circumferential resections in the sheep model

\begin{tabular}{|c|c|c|c|c|}
\hline $\begin{array}{l}\text { Length of } \\
\text { resection } \mathrm{cm}\end{array}$ & $n$ & $\begin{array}{l}\text { Time to complete mucosal } \\
\text { repair }{ }^{\mathrm{a}} \text { months/animal mean } \\
\text { (range) }\end{array}$ & $\begin{array}{l}\text { Mean nb dilatations } \\
\text { at final follow-up nb/animal } \\
\text { mean (range) }\end{array}$ & $\begin{array}{l}\text { Stenosis at final } \\
\text { follow-up nb }\end{array}$ \\
\hline 2.2 & 6 & 3.0 (all) & $1.3(0-3)$ & 0 \\
\hline 3.3 & 6 & $4.0(3-5)$ & $2.0(0-5)$ & 0 \\
\hline 4.4 & 7 & $3.4(3-4)$ & $1.4(0-2)$ & 0 \\
\hline 5.5 & 5 & $4.8(3-7)$ & $2.6(0-5)$ & 1 \\
\hline
\end{tabular}

Both total number of dilatations and duration of the follow-up correlate with the length of resection.

${ }^{\text {a }}$ Follow-up endoscopies were carried out monthly until complete reepithelialization was observed. The sole stenosis (11 mm in diameter) remaining at the end of the follow-up occurred in a $5.5 \mathrm{~cm}$-long resection 
Grosjean et al. obtained a 32\% incidence of second primary cancer according to the histological criteria of Warren and Gates [5, 20]. The routine use of panendoscopy allows for the diagnosis of synchronous SCC at an early stage, which statistically improves the survival of these patients [5]. In turn, the use of abrasive sponge cytology in the esophagus during the follow-up of head and neck cancer patients results in the early detection of metachronous SCC of the digestive tract [10]. Small superficial SCC of the esophagus can be treated by current techniques of EMR. When the patient presents with a "field cancerization" of the hypopharynx and/or esophagus, extended or circumferential EMR may be a good therapeutic option. After delineation of the lesions (including occult HGIN or carcinoma) with Toluidine blue staining [7], extended EMR will provide specimen for an accurate microscopic staging, and even treat the patient. Photodynamic therapy can be envisaged for multiple primaries or field cancerization, but the in-depth necrosis is variable and histological analysis fails [16]. However, these individuals most often undergo a pharyngoesophagectomy or an endoscopic brachytherapy because of the lack of other treatment modalities. Squamous cell carcinomas of the piriform sinus can also be treated by transoral $\mathrm{CO} 2$ laser resections with high rates of organ-preservation [18], but for superficial microinvasive carcinoma, EMR with the rigid esophagoscope will be simpler and much faster.

\section{Rigid endoscope for mucosectomy}

Our studies indicate that the modified rigid endoscope offers the possibility of achieving long and/or circumferential resections. Each resection provides a single rectangular specimen of $2-12 \mathrm{~cm}^{2}$ in size. Only two resections are needed for a $360^{\circ}$ EMR in the esophagus. The total surface of resected mucosa corresponds to the usual extension of a circumferential BE of up to $6 \mathrm{~cm}$ in length. The specimens are easily adjustable by the endoscopist and are thus adequate for histological analysis. The depth of resection $(1.0 \pm 0.1 \mathrm{~mm})$ is precisely situated at the level of the submucosa along the whole specimen. This allows a complete removal of dysplastic lesions, early SCC or AC confined to the mucosa (T1a cancer). Moreover, the deep glands found in $\mathrm{BE}$ will also be treated since the mean depth of metaplastic mucosa is estimated from pathological studies to be $0.5 \mathrm{~mm}$ (range: 0.39 to $0.59 \mathrm{~mm}$ ) [1].

The safety of the procedure has been demonstrated by our successive investigations in the sheep model, showing very low perforation rates $[4,11,12]$. No perforation occurred when only hemi-circumferential resections were performed. The main risk of circumferential EMR is the overlap of the two adjacent resections ( 1 case out of 24 circumferential resections), which should normally be avoided by submucosal injection of saline and visual control prior to the resection.

The EMR-rigid esophagoscope is currently the only instrument that allows the resection of a large mucosal specimens (up to $12 \mathrm{~cm}^{2}$ ) in a single piece and at a constant and predetermined depth. These characteristics encourage clinical applications such as the precise staging of early cancers of the upper digestive tract, the curative treatment of early SCC or AC (up to stage T1a or m3) [2] of the esophagus and early SCC (in situ or microinvasive) of the hypopharynx.

Further investigations in humans are now approved by the ethics commitee of our institution and will first attempt at hemi-circumferential resections for the treatment of early SCC of the hypopharynx and esophagus or for incomplete $\mathrm{BE}$, probably as tongues or flame-like projections from the Z-line.

\section{References}

1. Ackroyd R, Brown NJ, Stephenson TJ, Stoddard CJ, Reed MWR (1999) Ablation treatment for Barrett esophagus: what depth of tissue destruction is needed? J Clin Pathol 52:509512

2. Eguchi T, Nakanishi Y, Shimoda T, Iwasaki M, Igaki H, Tachimori Y, Kato H, Yamaguchi H, Saito D, Umemura S (2006) Histopathological criteria for additional treatment after endoscopic mucosal resection for esophageal cancer: analysis of 464 surgically resected cases. Mod Pathol 19:475-480

3. Giovannini M, Bories E, Pesenti C, Moutardier V, Monges G, Danisi C, Lelong B, Delpro JR (2004) Circumferential endoscopic mucosal resection in Barrett's esophagus with highgrade intraepithelial neoplasia or mucosal cancer. Preliminary results in 21 patients. Endoscopy 36:782-787

4. Grosjean P (1998) La mucosectomie endoscopique oesophagienne: étude pré-clinique. Akt Prob Otolaryngol 21:293302

5. Grosjean P, Monnier P (2000) Impact of the detection and treatment of early-stage second primary tumours on the outcome of patients with head and neck cancer. Schweitz Med Wochenschr 130:43S-46S

6. Kida M (2000) Endoscopic tumor diagnosis and treatment. Endoscopy 32:836-844

7. Monnier P, Savary M, Pasche R (1981) Contribution of toluidine blue to bucco-pharyngo-esophageal cancerology. Acta Endoscopica 11:299-315

8. Nigro JJ, Hagen JA, DeMeester TR, DeMeester SR, Peters JH, Oberg S, Theisen J, Kiyabu M, Crookes PF, Bremner CG (1999) Prevalence and location of nodal metastases in distal esophageal adenocarcinoma confined to the wall: implications for therapy. J Thorac Cardiovasc Surg 117:16-25

9. Nigro JJ, Hagen JA, DeMeester TR, DeMeester SR, Theisen J, Peters JH, Kiyabu M (1999) Occult esophageal adenocarcinoma: extent of disease and implications for effective therapy. Ann Surg 230:433-440 
10. Pellanda A, Grosjean $\mathrm{P}$, Leoni S, Mihaescu A, Monnier P, Pasche P (1999) Abrasive esophageal cytology for the oncological follow-up of patients with head and neck cancer. Laryngoscope 109:1703-1708

11. Pilloud R, Jaquet Y, Monnier P (2005) Extensive circumferential endoscopic mucosal resection with a new rigid esophagoscope: an animal study. J Thorac Cardiovasc Surg 130:1399-1405

12. Radu A, Grosjean P, Fontolliet C, Monnier P (2004) Endoscopic mucosal resection in the esophagus with a new rigid device: an animal study. Endoscopy 36:298-305

13. Rajan E, Gostout CJ, Feitoza AB, Leontovich ON, Herman LJ, Burgart LJ, Chung S, Cotton PB, Hawes RH, Kalloo AN, Kantsevoy SV, Pasricha PJ (2004) Widespread EMR: a new technique for removal of large areas of mucosa. Gastrointest endosc 60:623-627

14. Rösch T, Sarbia M, Schumacher B, Deinert K, Frimberg E, Troemer T, Stolte M, Neuhaus H (2004) Attempted endoscopic en bloc resection of mucosal and submucosal tumors using insulated-tips knives: a pilot series. Endoscopy 36:788-801

15. Satodate H, Inoue H, Yoshida T, Usui S, Iwashita M, Fukami N, Shiokawa A, Kudo SE (2003) Circumferential EMR of carcinoma arising in Barrett's esophagus: case report. Gastrointest Endosc 58:288-292

16. Savary J-F, Grosjean P, Monnier P, Fontolliet C, Wagnières G, Braichotte D, van den Bergh H (1998) Photodynamic therapy of early squamous cell carcinomas of the esophagus: a review of 31 cases. Endoscopy 30:258-265

17. Seewald S, Akaraviputh T, Seitz U, Brand B, Groth S, Mendoza G (2003) Circumferential EMR and complete removal of Barrett's epithelium: a new approach to management of Barrett's esophagus containing high-grade intraepithelial neoplasia and intramucosal carcinoma. Gastrointest Endosc 57:854-859

18. Steiner W, Ambrosch P, Hess CF, Kron M (2001) Organ preservation by transoral laser microsurgery in piriform sinus carcinoma. Otolaryngol Head Neck Surg 124:58-67

19. Stoeckli SJ, Zimmerman R, Schmidt S (2001) Role of routine panendoscopy in cancer of the upper aerodigestive tract. Otolaryngol Head Neck Surg 124:208-212

20. Warren S, Gates O (1932) Multiple primary malignant tumors: a survey of the literature and statistical study. Am J Cancer 51:1358-1403 